\title{
CENTER VORTEX MODEL FOR NONPERTURBATIVE STRONG INTERACTION PHYSICS
}

\author{
MICHAEL ENGELHARDT* \\ Institut für Theoretische Physik, Universität Tübingen \\ Auf der Morgenstelle 14, 72076 Tübingen, Germany
}

\begin{abstract}
A model for the infrared sector of $S U(2)$ Yang-Mills theory, based on magnetic vortex degrees of freedom represented by (closed) random world-surfaces, is presented. The model quantitatively describes both the confinement properties (including the finitetemperature transition to a deconfined phase) and the topological susceptibility of the Yang-Mills ensemble. A (quenched) study of the spectrum of the Dirac operator furthermore yields a behavior for the chiral condensate which is compatible with results obtained in lattice gauge theory.
\end{abstract}

\section{Introduction}

Strong interaction physics is characterized by diverse nonperturbative phenomena. Color charge is confined, chiral symmetry is spontaneously broken, and the axial $U(1)$ part of the flavor symmetry exhibits an anomaly. Moreover, at finite temperatures, one expects to encounter a deconfining phase transition. In principle, a theoretical tool exists which permits the calculation of any observable associated with these phenomena, namely lattice gauge theory. Nevertheless, it is useful to concomitantly formulate effective models which concentrate on the relevant infrared degrees of freedom, and thus provide a clearer picture of the dominant physical mechanisms. This facilitates the exploration of problem areas which are difficult to access using the full (lattice) gauge theory. The vortex model [1]-[4] presented in the following aims to provide a comprehensive quantitative description of all of the aforementioned nonperturbative aspects of the strong interaction within a unified, consistent framework. This turns out to be possible on the basis of a simple effective model dynamics; namely, vortex world-surfaces can be regarded as random surfaces on large length scales.

\footnotetext{
* Supported by DFG under grants Re 856/4-1 and Al 279/3-3.
} 


\section{Chromomagnetic center vortices and their dynamics}

Center vortices are closed lines of chromomagnetic flux in three-dimensional space; thus, they are described by closed two-dimensional world-surfaces in four-dimensional space-time. Their magnetic flux is quantized such that they contribute a phase corresponding to a nontrivial center element of the gauge group to any Wilson loop they are linked to (or, equivalently, whose minimal area they pierce). In the case of $S U(2)$ color discussed here, the only such nontrivial center phase is $(-1)$. For higher gauge groups, one must consider several possible fluxes carried by vortices.

As an illustrative example, consider a vortex surface located at a fixed point on the 1-2 plane, i.e. extending into the 3 and 4 directions. Such a vortex surface can be associated with a gauge field which depends only on the $x_{1}$ and $x_{2}$ coordinates as indicated in Fig. 1.

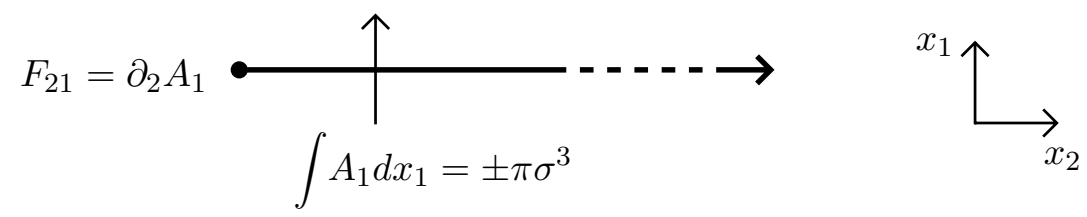

Figure 1. Local construction of a gauge field describing a vortex. Only the 1-2 plane is depicted; the vortex world-surface is a sheet extending into the 3 and 4 directions. In the 1-2 plane, it therefore shows up as a point. The vortex surface can be viewed as the boundary of a three-dimensional volume in four-dimensional space-time; in the two-dimensional slice of space-time depicted, this volume shows up as a line emanating from the vortex. The volume can be used to define the vortex gauge field: The latter shall have support precisely on the volume (i.e. be described by a $\delta$-function in the coordinate locally perpendicular to the volume); as a Lorentz vector, it shall point locally perpendicular to the volume, it shall point in 3-direction in color space (as encoded in the third Pauli matrix $\sigma^{3}$ ), and it shall have a magnitude such that the line integral shown in the figure acquires the specified value. Evaluating a Wilson loop enclosing the position of the vortex then yields the value -1 , as required. The field strength of the vortex is localized on the vortex; its only nonvanishing tensor component is the one associated with the two space-time directions locally perpendicular to the vortex surface. Note that there is a free choice of sign of the gauge field, corresponding to the two possible orientations of the vortex flux. Note also that, globally, the gauge field support line in the figure must end at another vortex elsewhere in the 1-2 plane; complications can arise if the vortex surfaces (and the three-dimensional volumes spanning them) are nonorientable. Such global issues are addressed in section $\mathrm{E}$.

Note that the vortex in Fig. 1 is infinitely thin, and the associated field strength is concentrated on the thin vortex world-surface. The real physical vortex fluxes conjectured to describe the infrared aspects of the Yang-Mills ensemble within the vortex picture of course should be thought 
of as possessing a finite thickness:; however, for the purpose of evaluating deeply infrared observables, i.e. looking from far away, the thin idealization is adequate. Nevertheless, the thickness of the vortices will significantly influence the ansatz for the vortex dynamics presented below, and the physical interpretation of that ansatz.

It should be remarked that the chromomagnetic vortex fluxes described above correspond to the flux domains found in the so-called Copenhagen vacuum [6]. Thus, the idea that these degrees of freedom may be relevant in the infrared sector of Yang-Mills theory is not new. What is new about the model to be discussed here is that the vortex picture has been developed into a quantitative tool, allowing to evaluate diverse physical observables, and that it furthermore has been generalized to finite temperatures, including the deconfined phase.

To arrive at a tractable dynamics for the model vortex world-surfaces, the latter will be composed of elementary squares (plaquettes) on a hypercubic lattice. The spacing of this lattice will be a fixed physical quantity related to the thickness of the vortex fluxes already mentioned further above; the lattice prevents an arbitrarily close packing of the vortices. Thus, it is not envisaged to eventually take the lattice spacing to zero, and accordingly renormalize the coupling constants, such as to arrive at a continuum theory. Rather, the lattice spacing represents the fixed physical cutoff one expects to be present in any infrared effective theory, and thus also delineates the ultraviolet limit of validity of the model. If one wants to refine the model such as to eliminate the artificial hypercubic nature of the vortex surfaces, one has to replace the lattice spacing by some other ultraviolet cutoff. For instance, if the surfaces are represented as triangulations, a minimal area of the elementary triangles could take on this role.

On the hypercubic lattice adopted here, the vortex surfaces will be regarded as random surfaces. These surfaces will be generated using Monte Carlo methods; the weight function specifying the ensemble depends on the curvature of the surfaces as follows [1]. Every instance of a link on the lattice being common to two plaquettes which are part of a vortex surface, but which do not lie in the same plane, is penalized by an action increment $c$. Thus, the action can be represented pictorially as

$$
S_{\text {curv }}=c \cdot \#(\square)
$$

Note that several such pairs of plaquettes can occur for any given link. E.g., if six vortex plaquettes are attached to a link, the action increment is $12 c$.

\footnotetext{
1 Phenomenologically, such a physical thickness has e.g. been argued to be crucial for an explanation of the Casimir scaling behavior of adjoint representation Wilson loops [5].
} 


\section{Confinement and Deconfinement}

Given the random surface dynamics defined in the previous section, it is now straightforward to evaluate Wilson loops, using the fundamental property of vortices that they modify any Wilson loop by a phase factor $(-1)$ whenever they pierce its minimal area. Such measurements can furthermore be carried out at several temperatures, by adjusting the extension of (Euclidean) space-time in the time direction; this extension is identified with the inverse temperature of the ensemble. At finite temperatures, static quark potentials are given by Polyakov loop correlators; their properties in the presence of vortex fluxes are completely analogous to the properties of Wilson loops. Qualitatively, as long as the curvature coefficient $c$ is not too large, one finds a confined phase (non-zero string tension) at low temperatures, and a phase transition to a high-temperature deconfined phase. In order to make the correspondence to full $S U(2)$ Yang-Mills theory quantitative, one can adjust $c$ such as to reproduce the ratio of the deconfinement temperature to the square root of the zero-temperature string tension, $T_{C} / \sqrt{\sigma_{0}}=0.69$. This happens at the value $c=0.24$. Furthermore, by setting $\sigma_{0}=(440 \mathrm{MeV})^{2}$ to fix the scale, one extracts from the measurement of $\sigma_{0} a^{2}$ the lattice spacing $a=0.39 \mathrm{fm}$. As discussed in the previous section, this is a fixed physical quantity, related to the thickness of the vortices, which represents the ultraviolet limit of validity of the effective vortex model.

Fig. 2 displays the results of string tension measurements on $16^{3} \times N_{t}$ lattices, as a function of temperature. After having fixed $c$ as described above, the so-called spatial string tension $\sigma_{s}$ can be predicted, cf. Fig. 2. In the high-temperature regime, it begins to rise with temperature; the value obtained at $T=1.67 T_{C}$, namely $\sigma_{s}\left(T=1.67 T_{C}\right)=1.39 \sigma_{0}$, corresponds to within $1 \%$ with the value measured in full $S U(2)$ Yang-Mills theory [7].

The confined and deconfined phases can alternatively be characterized by the percolation properties [1] of the vortex clusters in space slices of the lattice universe, i.e. slices defined by keeping one space coordinate fixed. In such a slice of space-time, vortices are represented by closed lines, cf. Fig. 3 . In the confined phase, these lines percolate throughout (sliced) space-time, whereas in the deconfined phase, they form small, isolated clusters, which, more specifically, wind around the universe in the Euclidean time direction (and are closed by virtue of the periodic boundary conditions). Also, simple heuristic arguments can be given [1] which explain why confinement should be associated with vortex percolation. The percolation characteristics of the surfaces in the vortex model closely mirror the ones found for vortex structures extracted from full lattice Yang-Mills configurations via an appropriate gauge fixing and projection procedure [8], [9], which were investigated in [10]. 


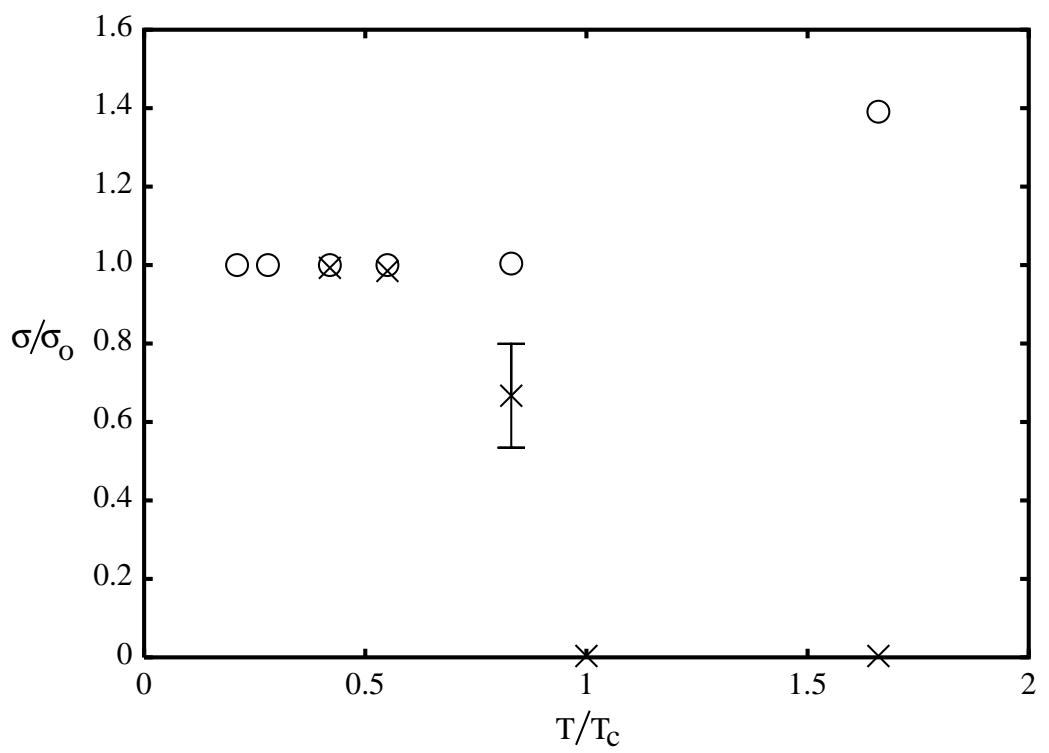

Figure 2. String tension between static color sources (crosses) and spatial string tension (circles) as a function of temperature, obtained with a curvature coefficient of $c=0.24$ on $16^{3} \times N_{t}$ lattices.

\section{Topology}

Besides confinement, the topological properties of the Yang-Mills ensemble constitute an important nonperturbative aspect of strong interaction theory. These properties are encoded in the topological charge

$$
Q=\frac{1}{32 \pi^{2}} \int d^{4} x \epsilon_{\mu \nu \lambda \tau} \operatorname{Tr} F_{\mu \nu} F_{\lambda \tau}
$$

In view of (21), nonvanishing topological density is generated at a given space-time point if the field strength there has nonvanishing tensor components such that the corresponding Lorentz indices span all four space-time directions. For example, a nonvanishing $F_{12}$ in conjunction with a nonvanishing $F_{34}$ fulfils this requirement. Nonvanishing $F_{12}$ arises when a vortex surface segment (locally) runs in 3-4 direction, as discussed in section 2; conversely, for nonvanishing $F_{34}$, one needs a surface segment running in 1-2 direction. Thus, a nontrivial contribution to the topological charge is e.g. generated by a self-intersection point of the vortex surfaces, where a segment running in 3-4 direction intersects a segment running in 1-2 direction. Quantitatively [11], the contribution specifically of such a self-intersection point is $\pm 1 / 2$. In general, all singular points of a surface configuration on the hypercubic lattice contribute to the topological charge, where a 


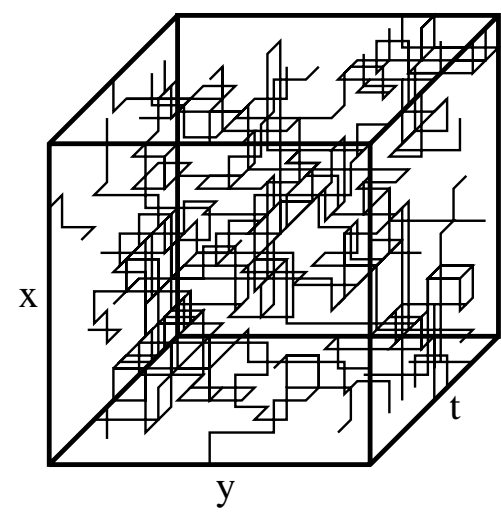

confined phase

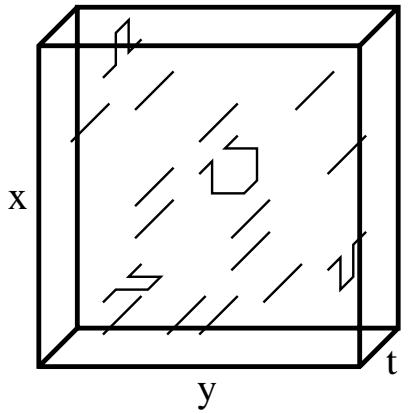

deconfined phase

Figure 3. Typical vortex configurations in the confined and the deconfined phases.

singular point is defined as a point (lattice site) at which the set of tangent vectors to the surface configuration spans all four space-time directions. Self-intersection points are but the simplest example of such singular points; there also exist writhings, at which the surface is twisted in such a way as to generate a singular point in the above sense. These writhings actually turn out to be statistically far more important than the self-intersection points in the random surface ensemble discussed here [2].

Contrary to the Wilson loop, the topological charge $Q$ is sensitive to the orientation of the vortex surfaces via the signs of the field strengths entering (2). The random surfaces of the vortex ensemble should not be thought of as being globally oriented (in fact, most configurations are not even globally orientable); they in general are composed of patches of alternating orientation. Given a vortex surface from the ensemble defined by (1), it is straightforward to furthermore randomly assign orientations to the plaquettes making up the surface. By biasing this procedure with respect to the relative orientation of neighboring plaquettes, different mean sizes of the oriented patches making up the surface can be generated; equivalently, the density of patch boundary lines can be adjusted. This density strictly speaking constitutes an additional parameter of the model, which cannot be fixed using the confinement properties due to the fact that the Wilson loop is insensitive to the vortex orientation. A priori, one might expect e.g. the topological susceptibility $\chi=\left\langle Q^{2}\right\rangle / V$, where $V$ denotes the spacetime volume under consideration, to depend on this parameter. This would mean that $\chi$ can possibly be fitted, but not predicted. In actual fact, it turns out that this quantity is independent of the aforementioned density within 


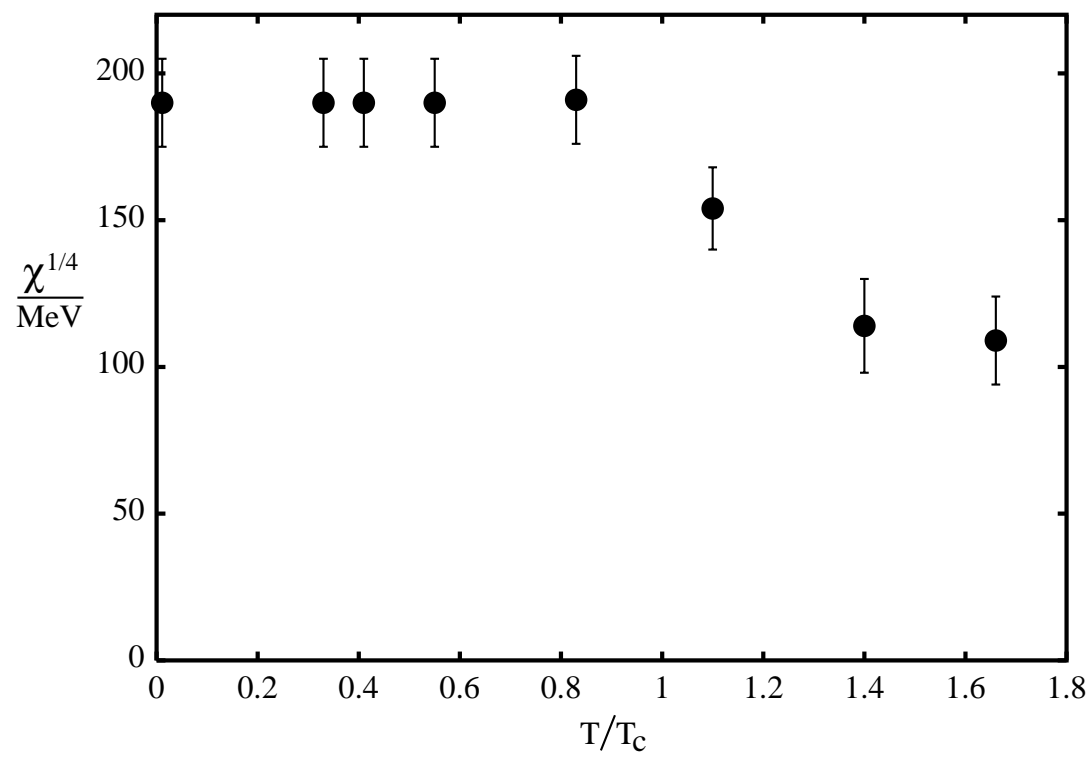

Figure 4. Fourth root of the topological susceptibility as a function of temperature, obtained on $12^{3} \times N_{t}$ lattices.

the error bars. The reasons for this can be understood in detail in terms of the geometrical properties of the random surfaces [2]. The measurement of the topological susceptibility $\chi$ discussed below thus does represent a genuine quantitative prediction of the vortex model.

In practice, before the topological charge can be extracted, in the manner indicated above, from the geometrical properties of the model hypercubic lattice surfaces, ambiguities must be resolved [2] which would not appear if one were dealing with arbitrary surfaces in continuous space-time, and which are reminiscent of the ones occuring in standard lattice gauge theory. Only after construction of an appropriate "inverse-blocking" algorithm, the topological charge $Q$ of the vortex surface configurations can be evaluated, allowing to determine the topological susceptibility of the vortex ensemble. The result is exhibited in Fig. 1 as a function of temperature.

Quantitatively, this result is compatible with measurements in full YangMills theory [12]. The vortex model thus provides, within one common framework, a simultaneous, consistent description of both the confinement properties as well as the topological properties of the $S U(2)$ Yang-Mills ensemble. It should be remarked that, also as far as the vortex structures extracted from lattice Yang-Mills configurations via center gauge fixing and center projection [8], [9] are concerned, evidence exists that these structures encode the topological characteristics of the gauge fields 13, 14. 


\section{Spontaneous chiral symmetry breaking}

A comprehensive description of the nonperturbative phenomena which determine strong interaction physics must furthermore include the coupling of the vortices to quark degrees of freedom and the associated spontaneous breaking of chiral symmetry. The latter can be quantified via the chiral condensate $\langle\bar{\psi} \psi\rangle$, which is related to the spectral density $\rho(\lambda)$ of the Dirac operator $I D$ in a vortex background via the Casher-Banks formula [15]

$$
\langle\bar{\psi} \psi\rangle=-\lim _{V \rightarrow \infty} 2 m \int_{0}^{\infty} d \lambda \frac{\rho(\lambda)}{m^{2}+\lambda^{2}}
$$

where $m$ denotes the quark mass and $V$ the space-time volume. In the chiral limit $m \rightarrow 0$, the chiral condensate thus behaves as $\langle\bar{\psi} \psi\rangle \rightarrow-\pi \rho(0)$.

In order to construct the Dirac operator, it is necessary to cast the vortex fluxes explicitly in terms of gauge fields. Locally, this can be achieved as displayed in Fig. 田, but globally, a difficulty arises: Generic vortex surfaces are not orientable. Viewing vortex surfaces as boundaries of three-volumes (which represent the support of the associated gauge field, cf. Fig. 1), also these three-volumes are in general not oriented. As a consequence, the gauge field either contains Dirac strings or must be defined on different space-time patches in the spirit of the Wu-Yang construction, cf. Fig. 5. In practice, the description via Dirac strings is not suitable, for the following reason: If one solved the Dirac equation in the presence of Dirac strings exactly, then the quark wave functions would exhibit singularities along the Dirac strings which would cancel any physical effect of these strings. The Dirac strings would be unobservable, as they should be. However, in a truncated calculation, employing only infrared quark modes, cf. below, the cancellation would not be perfect; instead, the Dirac strings effectively would act as additional physical magnetic fluxes (of magnitude double that of vortex fluxes) with which the quarks can interact. Thus, more magnetic disorder would in effect be present than the model aims to describe. Consequently, it is more appropriate to use the $\mathrm{Wu}$-Yang construction for the gauge field. On the individual space-time patches, which must be chosen sufficiently small, the vortices are orientable and no Dirac strings arise. The patches then are related by transition functions, which are chosen non-Abelian, cf. Fig. 5 .

Having defined an explicit gauge field representation of the vortex configurations, one can evaluate (analytically) all matrix elements of the Dirac operator in a truncated, infrared basis of quark wave functions. This matrix representation of the Dirac operator then determines the propagation of the infrared quark modes spanned by the basis. The basis used in the present work is of the finite element type [4]; for each $2^{4}$ cube in the hypercubic lattice on which the vortex surfaces are defined, there is a quark basis 

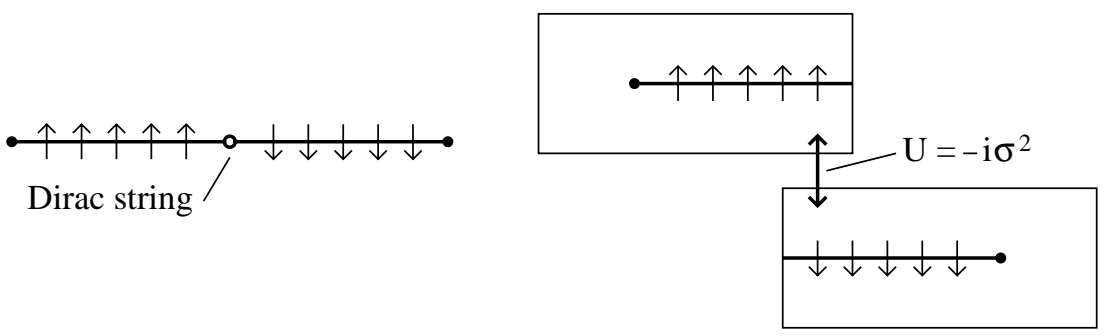

Figure 5. Nonoriented gauge field support volume in a two-dimensional slice of space-time. In such a slice, vortices show up as the solid points, volumes emanating from them as the solid lines. The directions of the vortex fluxes at the boundaries of the volumes, fixed a priori, here happen to be such that the volume segments emanating from the different vortices are forced to have mutually incompatible orientation. This is indicated by the arrows, which symbolize (directed) line integrals $\int A_{\mu} d x_{\mu}$; all of the line integrals indicated in the figures yield the same value, for definiteness $+\pi \sigma^{3}$. Left: Attempt at a global definition of the gauge field; the gauge field support volume then necessarily contains the indicated Dirac string, carrying flux double that of a vortex. Right: Wu-Yang construction, with space-time subdivided into two patches, on each of which the volume is oriented. Gauge fields on the two patches are related by the transition function $U$. In the simple example depicted here, $U$ can be chosen constant in the whole overlap region shared by the two patches. In generic configurations, the presence of other vortices nearby forces the space-time region of nontrivial $U$ to be more localized, namely onto the immediate vicinity of the gauge field support volume. For further details on this point and the complications it entails, cf. [4].

function which is localized on that cube (and which is piecewise linear in each of the space-time coordinates). These $2^{4}$ cubes serve a dual purpose; they at the same time represent the space-time patches which are used in practice to define the vortex gauge field as discussed further above.

Before turning to the numerical results, note that some options still remain within the above construction scheme for the gauge field: Since the gauge field support three-volume only must satisfy the property that its boundary reproduces the locations of the vortex fluxes, one can freely choose its interior. It can be constructed in a random fashion, which will yield a rather rough three-volume, or in a smoothed fashion. Likewise, when defining the gauge fields on the individual space-time patches, one can choose a procedure which is not biased with respect to the relative color orientations of adjacent patches; such a random relative color orientation will yield a high density of nontrivial transition functions. Alternatively, one can consider supplementing this by (gauge) transformations $A \rightarrow-A$ on individual patches such as to align their color orientations with those of neighboring patches, leading to maximally smooth transition functions.

Having constructed a finite matrix representation of the Dirac operator 


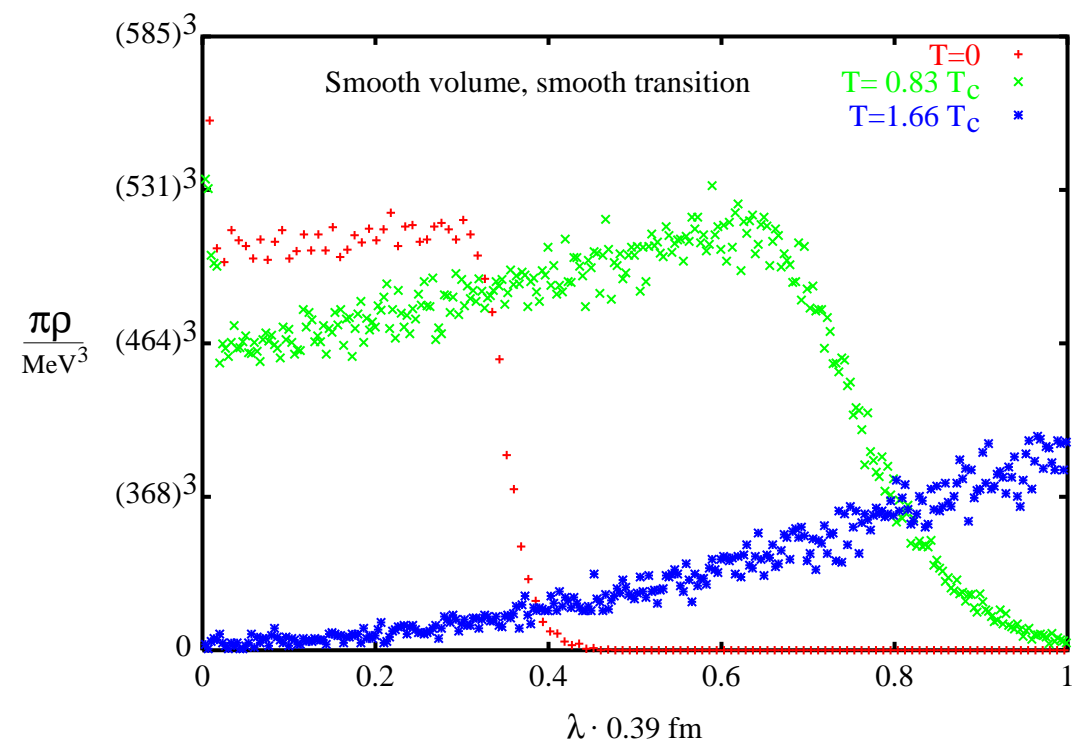

Figure 6. Dirac spectral density at different temperatures for the maximally smooth construction of the vortex gauge field, obtained using a spatial extension of the lattice of $4 a=1.56 \mathrm{fm}$.

in the above fashion, one can numerically evaluate the (vortex ensemble average of the) Dirac spectral density $\rho(\lambda)$. The results for the maximally smooth gauge field construction (in the sense of the previous paragraph) are depicted in Fig. 6. The qualitative properties of the spectrum of the Dirac operator are as follows. At very small eigenvalues $\lambda$, there is an anomalous enhancement of the spectral density which is presumably due to the quenched approximation used here; a similar effect has e.g. been argued to occur in quenched chiral perturbation theory [16] and may contribute to the divergent chiral condensate in the chiral limit observed in lattice calculations employing domain wall fermions [17]. Apart from this divergence, the bulk of the spectrum, which for sufficiently large quark masses dominates the value of the chiral condensate, cf. eq. (3), extrapolates to a finite value at $\lambda=0$ in the confining phase; this signals the spontaneous breaking of chiral symmetry. Quantitatively, the zero-temperature chiral condensate extracted by extrapolating the bulk of the spectrum to zero eigenvalue $\lambda$, cf. Fig. 6, is of the same order of magnitude as the one found in lattice Yang-Mills theory [18], which amounts to $-(450 \mathrm{MeV})^{3}$ (the scale having been fixed in the same way as in the present model). This result is reasonably robust if one uses rougher gauge field constructions (in the sense discussed above) [4]; the result for the zero-temperature chiral condensate then varies between $-(465 \mathrm{MeV})^{3}$ and $-(560 \mathrm{MeV})^{3}$. It should 
be noted that the actual value of the condensate by itself carries no direct physical meaning, since it is not a renormalization group invariant; only its product with the quark mass (which is as yet undefined within the model) is. However, the order of magnitude agreement with lattice gauge theory indicates that the vortex model yields a natural description of spontaneous chiral symmetry breaking, without requiring e.g. unnaturally large or small quark masses. More detailed insight into the quark physics induced by the vortex ensemble will require the evaluation of further hadronic observables.

Turning to the deconfined phase, the differences between the various model options for the gauge field mentioned further above by contrast already become apparent at the qualitative level. The smoothest option, which is displayed in Fig. 6, is the only one which reproduces the behavior found in lattice gauge theory, namely a rapid drop in the chiral condensate as the temperature is raised above the deconfining phase transition. This appears to represent the model of choice. All other, rougher, gauge field models [if retain a substantial chiral condensate in the deconfined phase. It thus seems crucial to remove, as much as possible, ultraviolet artefacts in the vortex gauge fields in order to correctly describe that phase.

\section{Outlook}

With the Dirac operator at hand, one can also envisage carrying out dynamical quark calculations within the vortex model, by reweighting the vortex ensemble with the Dirac operator determinant. This will substantially penalize Dirac eigenvalues of very small magnitude and thus presumably reduce the chiral condensate to the phenomenologically expected values around $-(230 \mathrm{MeV})^{3}$. Moreover, to make contact with phenomenology, the vortex model must still be extended to $S U(3)$ color; the corresponding random surface ensemble will in some respects be qualitatively different from the one discussed here, since there are two nontrivial center elements in the $S U(3)$ group, namely the phases $e^{ \pm i 2 \pi / 3}$. One therefore must allow for two distinct vortex fluxes, which can branch and fuse into one another. This difference in the topological character of the configurations is e.g. expected to lead to a change in the order of the deconfinement phase transition from second order for $S U(2)$ to first order for $S U(3)$, as observed in full YangMills lattice experiments [19. Ultimately, it is hoped that this model will become a useful tool for phenomenological considerations.

\section{References}

1. Engelhardt, M. and Reinhardt, H. (2000) Center vortex model for the infrared sector of Yang-Mills theory - confinement and deconfinement, Nuclear Physics, B585, pp. 591-613 
2. Engelhardt, M. (2000) Center vortex model for the infrared sector of Yang-Mills theory - topological susceptibility, Nuclear Physics, B585, pp. 614-633

3. Engelhardt, M. (2002) Center vortex model for the infrared sector of Yang-Mills theory, Nuclear Physics (Proceedings Supplements), 106, pp. 655-657

4. Engelhardt, M. (2002) Center vortex model for the infrared sector of Yang-Mills theory - quenched Dirac spectrum and chiral condensate, hep-lat/0204002, pp. 1-35

5. Faber, M., Greensite, J. and Olejník, Š. (1998) Casimir scaling from center vortices: Towards an understanding of the adjoint string tension, Physical Review, D57, pp. 2603-2609

6. Nielsen, H.B. and Olesen, P. (1979) A quantum liquid model for the QCD vacuum: Gauge and rotational invariance of domained and quantized homogeneous color fields, Nuclear Physics, B160, pp. 380-396

Ambjørn, J. and Olesen, P. (1980) On the formation of a random color magnetic quantum liquid in QCD, Nuclear Physics, B170, pp. 60-78

7. Bali, G.S., Fingberg, J., Heller, U.M., Karsch, F. and Schilling, K. (1993) The spatial string tension in the deconfined phase of the (3+1)-dimensional $\mathrm{SU}(2)$ gauge theory, Physical Review Letters, 71, pp. 3059-3062

8. Del Debbio, L., Faber, M., Greensite, J. and Olejník, Š. (1997) Center dominance and Z(2) vortices in SU(2) lattice gauge theory, Physical Review, D55, pp. 2298-2306

9. Del Debbio, L., Faber, M., Giedt, J., Greensite, J. and Olejník, Š. (1998) Detection of center vortices in the lattice Yang-Mills vacuum, Physical Review, D58, pp. 094501$1-15$

10. Engelhardt, M., Langfeld, K., Reinhardt, H. and Tennert, O. (2000) Deconfinement in SU(2) Yang-Mills theory as a center vortex percolation transition, Physical Review, D61, pp. 054504-1-10

11. Engelhardt, M. and Reinhardt, H. (2000) Center projection vortices in continuum Yang-Mills theory, Nuclear Physics, B567, pp. 249-292

12. Allés, B., D'Elia, M. and Di Giacomo, A. (1997) Topology at zero and finite T in SU(2) Yang-Mills theory, Physics Letters, B412, pp. 119-124

13. De Forcrand, P. and D'Elia, M. (1999) On the relevance of center vortices to QCD, Physical Review Letters, 82, pp. 4582-4585

14. Bertle, R., Engelhardt, M. and Faber, M. (2001) Topological susceptibility of YangMills center projection vortices, Physical Review, D64, pp. 074504-1-10

15. Banks, T. and Casher, A. (1980) Chiral symmetry breaking in confining theories, Nuclear Physics, B169, pp. 103-125

16. Bernard, C. and Golterman, M. (1992) Chiral perturbation theory for the quenched approximation, Nuclear Physics (Proceedings Supplements), 26, pp. 360-362

Sharpe, S.R. (1993) Problems with the quenched approximation in the chiral limit, Nuclear Physics (Proceedings Supplements), 30, pp. 213-216

17. Fleming, G.T., Chen, P., Christ, N.H., Kaehler, A.L., Malureanu, C.I., Mawhinney, R.D., Siegert, G.U., Sui, C.-Z., Vranas, P.M. and Zhestkov, Y. (1999) The domain wall fermion chiral condensate in quenched QCD, Nuclear Physics (Proceedings Supplements), 73, pp. 207-209

18. Hands, S.J. and Teper, M. (1990) On the value and the origin of the chiral condensate in quenched SU(2) lattice gauge theory, Nuclear Physics, B347, pp. 819-853

19. Kogut, J.B., Polonyi, J., Wyld, H.W., Shigemitsu, J. and Sinclair, D.K. (1985) Further evidence for the first order nature of the pure gauge SU(3) deconfinement transition, Nuclear Physics, B251, pp. 311-332 